J. Clin. Chem. Clin. Biochem.

Vol. 16,1978 , pp. $525-529$

\title{
Induktion der Tyrosin-Aminotransferase (EC 2.5.1.5) in der Rattenleber durch Nicotinsäureamid
}

\author{
Von H. Kröger und Regina Grätz \\ Robert Koch-Institut des Bundesgesundheitsamtes, Abt. Biochemie, Berlin
}

(Eingegangen am 13. Juni/16. August 1978)

Herrn Prof. Dr. Dr. E. Schütte zum 70. Geburtstag gewidmet

Zusammenfassung: In intakten Ratten bewirkt Nicotinsäureamid einen Anstieg der Tyrosin-Aminotransferase in Abhängigkeit von der Dosis an Nicotinsäureamid. In adrenalektomierten Tieren steigt die Aktivität des Enzyms nur an bis zu einer Dosis von $250 \mathrm{mg} / \mathrm{kg}$ Nicotinsäureamid. Eine Kombination von Nicotinsäureamid mit $L$-Methionin und/ oder Cortisonacetat fuihrt kaum zu weiterem Enzym-Anstieg; erst bei zusätzlicher Gabe von $L$-Tyrosin wird dieser bemerkenswert.

\section{Induction of tyrosine aminotransferase (EC 2.5.1.5) by nicotinamide in rat liver}

Summary: In intact rats nicotinamide induces an increase of tyrosine aminotransferase depending on the dose of nicotinamide. In adrenalectomized rats an increase of tyrosine aminotransferase activity is only found up to a dose of $250 \mathrm{mg} / \mathrm{kg}$ nicotinamide. The combination of nicotinamide with $L$-methionine and/or cortisone acetate does not cause a significant increase of the enzyme activity, which, however, can be seen in the presence of $L$-tyrosine.

\section{Einführung}

In einer vorangehenden Arbeit befaßten wir uns mit dem Einfluß von $L$-Tryptophan auf die Induktion der Tyrosin-Aminotransferase (1). Wir beobachteten, daß die Induzierbarkeit der Tyrosin-Aminotransferase durch kleine Dosen Cortisonacetat und $L$-Tyrosin mit $L$-Tryptophan erheblich gefördert wird. In diesem Zusammenhang konnten wir auch die von Rosen et al. (2) beobachtete Induktions-Steigerung durch $L$-Methionin bestätigen. In den Rahmen dieser Studien haben wir nun dạs Nicotinsäureamid einbezogen. Von Blake und Kun (3-5) sowie Yamaguchi et al. (6) konnte bereits gezeigt werden, daß die Tyrosin-Aminotransferase durch Nicotinsäureamid induziert wird. Auch andere Enzyme werden durch das Nicotinsäureamid in ihrer Aktivität vermehrt (5-9). In der vorliegenden Arbeit haben. wir uns eingehend mit der Wirkung von Nicotinsäureamid auf die Aktivität der Tyrosin-Aminotransferase auseinandergesetzt.

\section{Material und Methoden}

Tiere

Für die Versuche wurden weibliche Ratten des Stammes Wistar (Zentrale Versuchstieraniage des Bundesgesundheitsamtes, Berlin) verwendet. Den adrenalektomierten Tieren wurde 5 Tage lang $0,15 \mathrm{~mol} / 1 \mathrm{NaCl}-L$ ösung gegeben; dann wurden sie für die
Versuche eingesetzt. Die Substanzen wurden den Tieren intraperitoneal appliziert. Die Kontrollen erhiel ten jeweils $10 \mathrm{ml} / \mathrm{kg}$ $0,15 \mathrm{~mol} / 1 \mathrm{NaCl}$-Lösung. In den Darstellungen ist der mittlere Fehler des Mittelwertes angegeben.

Bestimmung der Tyrosin-Aminotransferase

Die Aktivität des Enzyms wurde ermittelt wie bereits beschrieben (10). Die Protein-Bestimmung erfolgte nach dem BiuretVerfahren (11).

Substanzen

Nicotinsäureamid, $L$-Methionin und $L$-Tyrosin: Merck, Darmstadt; Cortisonacetat: Ciba, Wehr/Baden.

Ergebnisse

Intakte Tiere

\section{Nicotinsäureamid, normales Futter}

Bei Verabreichung von steigenden Dosen Nicotinsäureamid kommt es zu einer steten Zunahme der TyrosinAminotransferase-Aktivität (Abb. 1); schon etwa $80 \mathrm{mg} / \mathrm{kg}$ Nicotinsäureamid haben einen Effekt. Es fällt auf, daß die Streuung relativ groß ist.

\section{Nicotinsäureamid, 18 Stunden Hunger}

Aus Abbildung 2 ist zu entnehmen, daß schon mit $250 \mathrm{mg} / \mathrm{kg}$ Nicotinsäureamid eine relativ hohe Tyrosin- 


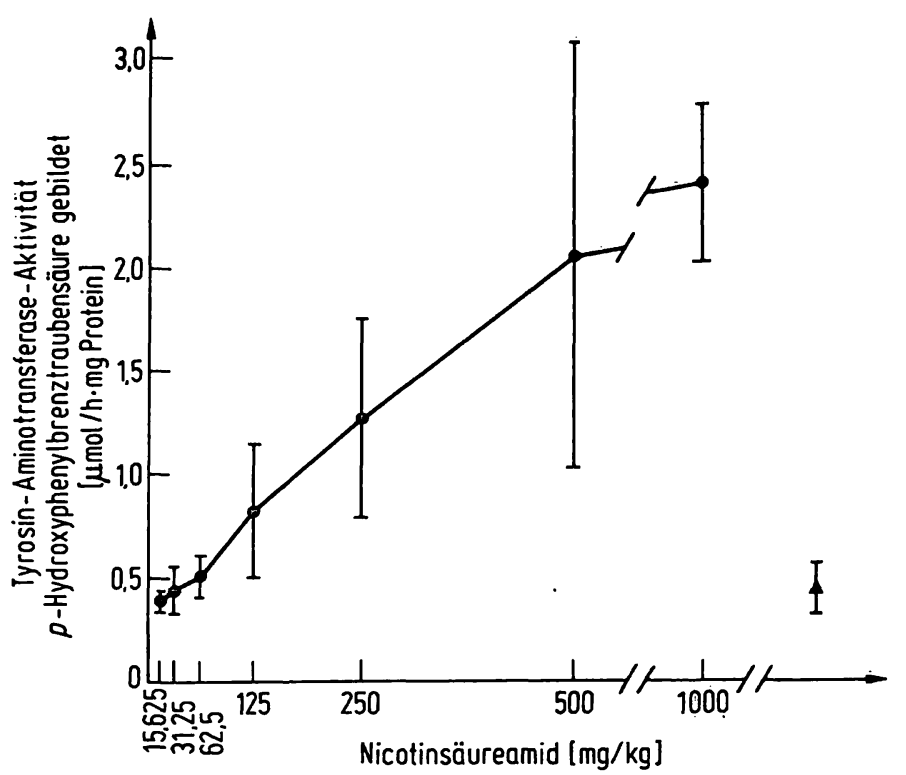

Abb. 1. Einfluß von steigenden Dosen Nicotinsäureamid auf die Aktivität der Tyrosin-Aminotransferase in der Leber normal ernährter, intakter Ratten.

Die Tiere wurden 9 Stunden nach der NicotinsäureamidInjektion getötet.

$\overline{\mathrm{x}} \pm s_{\overline{\mathrm{x}}} ; \mathrm{n}=\mathrm{je} 4-5$ Tiere

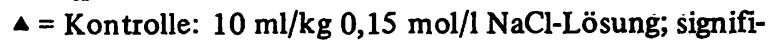
kant unterschiedlich bei $5 \%$ Irrtumswahrscheinlichkeit $\mathrm{ab} \geqq 125 \mathrm{mg} / \mathrm{kg}$ Nicotinsäureamid

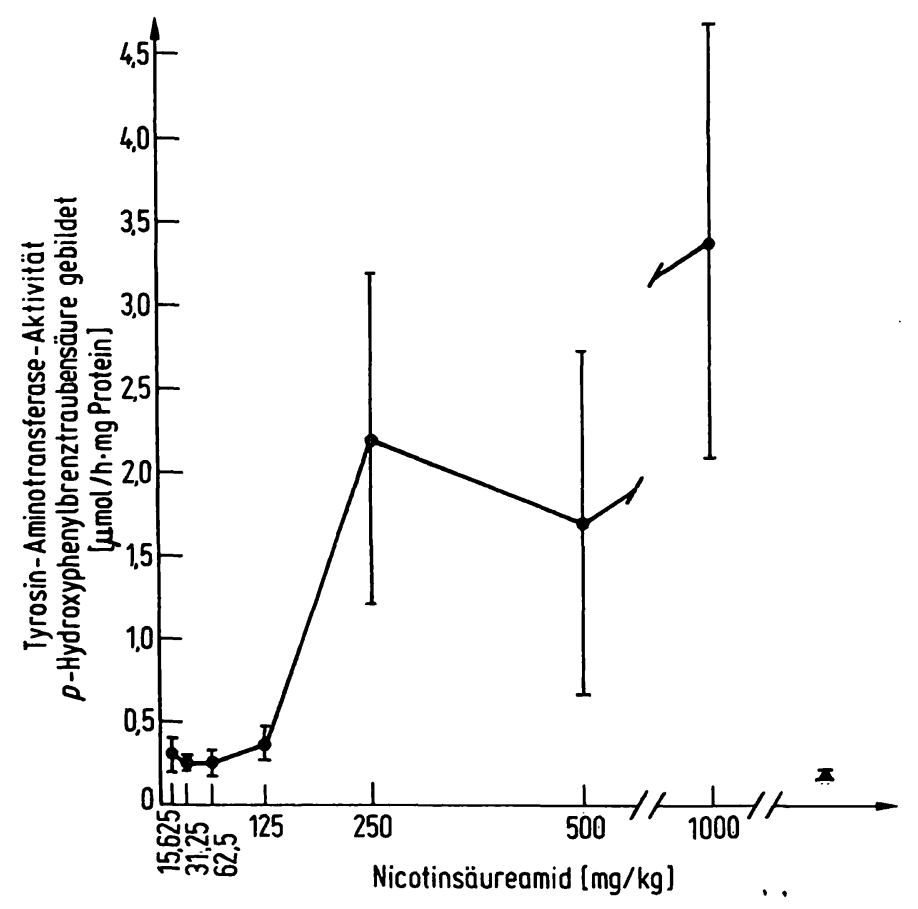

Abb. 2. Einfluß von steigenden Dosen Nicotinsäureamid auf die Ak tivität der Tyrosin-Aminotransferase in der Leber intakter Ratten nach 18 Stunden Hunger. Die Tiere wurden 9 Stunden nach der Nicotinsäureamid-Injektion getötet. $\overline{\mathrm{x}} \pm s_{\overline{\mathrm{x}}} ; \mathrm{n}=\mathrm{je}$ 5-6 Tiere

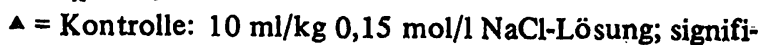
kant unterschiedlich bei $5 \%$ Irrtumswahrscheinlichkeit $\mathrm{ab} \geqq 250 \mathrm{mg} / \mathrm{kg}$ Nicotinsäureamid
Aminotransferase-Aktivität erzielt werden kann. Auch hier zeigt sich wieder eine erhebliche Streubreite.

\section{Adrenalek tomierte Tịere \\ Nicotinsäureamid, normales Futter}

Dosis-Abhängigkeit: Bei Gaben bis zu $250 \mathrm{mg} / \mathrm{kg}$ Nicotinsäureamid steigt die Aktivität der TyrosinAminotransferase in adrenalektomierten Tieren an (Abb. 3). Im Gegensatz zu den intakten Tieren ist die Aktivität jedoch geringer.

Zeit-Abhängigkeit: Bei Applikation von $500 \mathrm{mg} / \mathrm{kg}$ Nicotinsäureamid steigt die Aktivität der TyrosinAminotransferase nach 5 Stunden an (Abb. 4). Nach 7 Stunden sind die höchsten Werte erreicht, die auch über die weitere Beobachtungszeit konstant bleiben.

\section{Nicotinsäureamid, 18 Stunden Hunger}

Wie aus Abbildung $5 \mathrm{zu}$ ersehen ist, kommt es nach Nicotinsäureamid-Gabe ähnlich wie bei intakten Tieren, die gehungert haben, zu einem abrupten Anstieg der Tyrosin-Aminotransferase-Aktivität. Jedoch wird eine bestimmte Aktivitäts-Höhe ähnlich wie bei den normal ernährten, adrenalektomierten Tieren nicht überschritten.

\section{Nicotinsäureamid in Kombination mit anderen Substanzen, normales Futter}

Cortisonacetat: Wie frühere Studien ergeben haben, bewirken $2,5 \mathrm{mg} / \mathrm{kg}$ Cortisonacetat keine Induktion der Tyrosin-Aminotransferase (12). Auch in Kombination

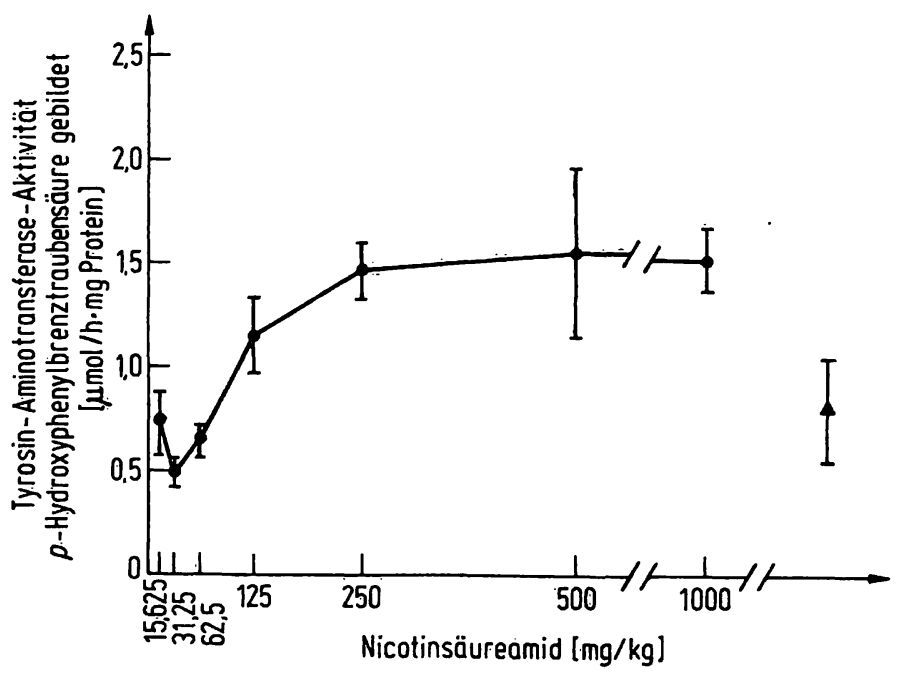

Abb. 3. Einfluß von steigenden Dosen Nicotinsäureamid auf die Aktivität der Tyrosin-Aminotransferase in der Leber normal ernährter, adrenalektomierter Ratten. Die Tiere wurden 9 Stunden nach der Nicotinsäureamid-Injektion getötet.

$\bar{x} \pm s_{\bar{x}} ; n=j . e 4$ Tiere

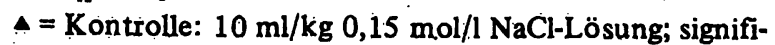
kant unterschiedlich bei $5 \%$ Irrtumswahrscheinlichkeit $\mathrm{ab} \geqq 250 \mathrm{mg} / \mathrm{kg}$ Nicọtinsäureamid 


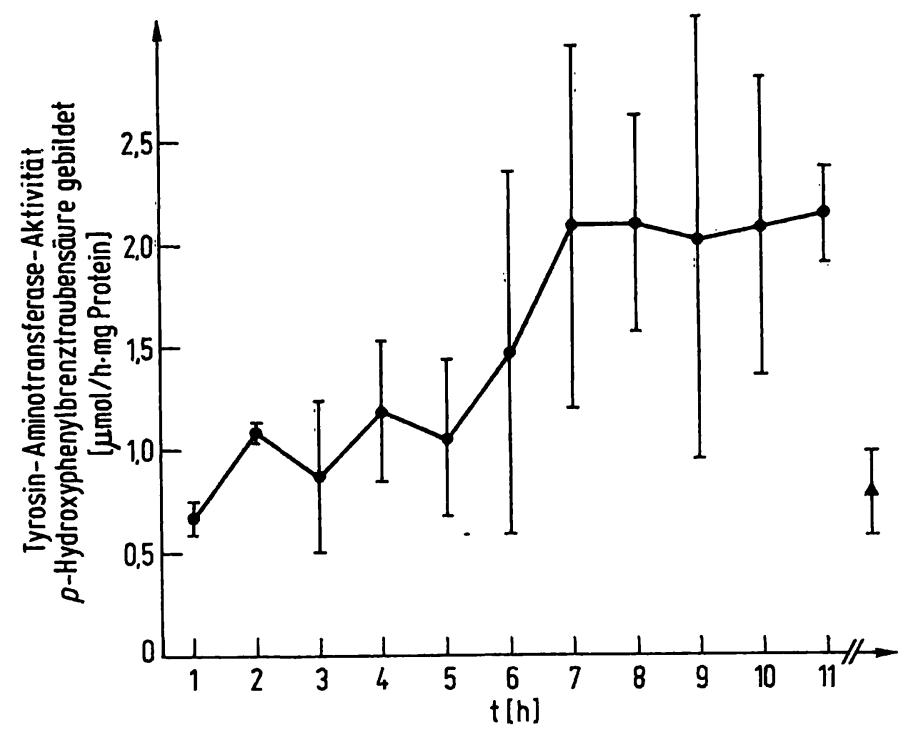

Abb. 4. Induktion der Tyrosin-Aminotransferase durch $500 \mathrm{mg} / \mathrm{kg}$ Nicotinsäureamid in der Leber normal ernährter, adrenalektomierter Ratten in Abhängigkeit von der Zeit.

$\overline{\mathbf{x}} \pm s_{\mathbf{x}} ; n=$ je 3 Tiere

$\Delta=$ Kontrolle: $10 \mathrm{ml} / \mathrm{kg}$ 0,15 mol/1 NaCl-Lösung; signifikant unterschiedlich bei $5 \%$ Irrtumswahrscheinlichkeit bei $2 \mathrm{~h}, 8 \mathrm{~h}, 10 \mathrm{~h}, 1 \mathrm{~h}$

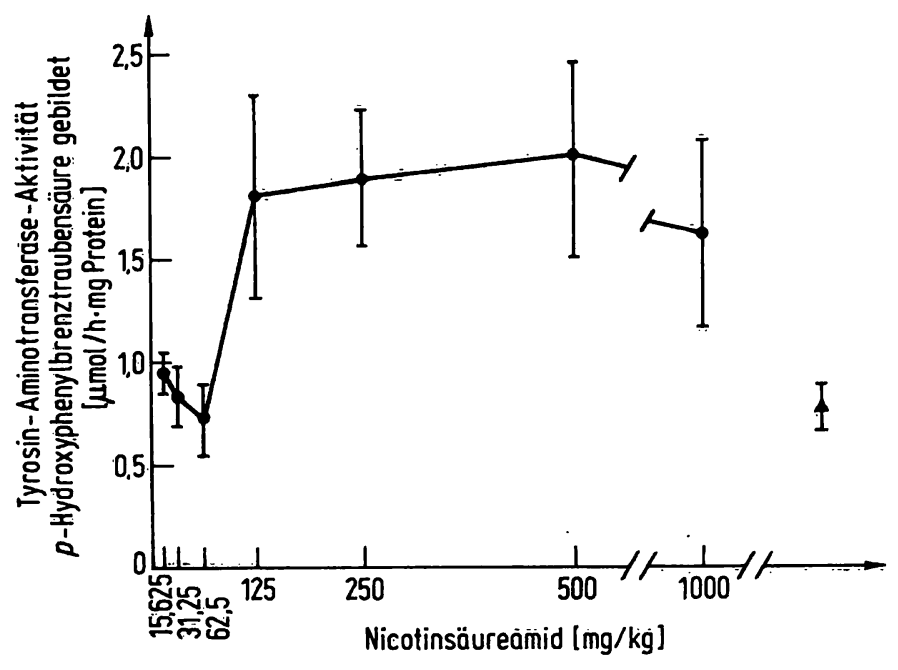

Abb. 5. Einfluß von steigenden Dosen Nicotinsäureamid auf die Aktivität der Tyrosin-Aminotransferase in der Leber adrenalek tomierter Ratten nach 18 Stunden Hunger. Die Tiere wurden 9 Stunden nach der Nicotinsäureamid-Injekktion getötet.

$\overline{\mathrm{x}} \pm s_{\overline{\mathrm{x}}} ; \mathrm{n}=$ je 5 Tiere

$\Delta=$ Kontrolle: $10 \mathrm{ml} / \mathrm{kg} 0,15 \mathrm{~mol} / \mathrm{l} \mathrm{NaCl}$-Lösung; signifikänt unterschiedlich bei $5 \%$ Irrtumswahrscheinlichkeit bei $15,625 \mathrm{mg} / \mathrm{kg}$ und $\mathrm{ab} \geqq 125 \mathrm{mg} / \mathrm{kg}$ Nicotinsäureamid

mit Nicotinsäureamid bleibt diesẹ Cortịsonacetat-Menge ohne Einfluß (Abb. 6).

L-Methionin (Zeit-Abhängigkeit): Aus Abbildung 7 ist zu entnehmen, daß bereits eine Stunde nach Applikation von $500 \mathrm{mg} / \mathrm{kg} L$-Methionin die Aktivität der Tyrosin-

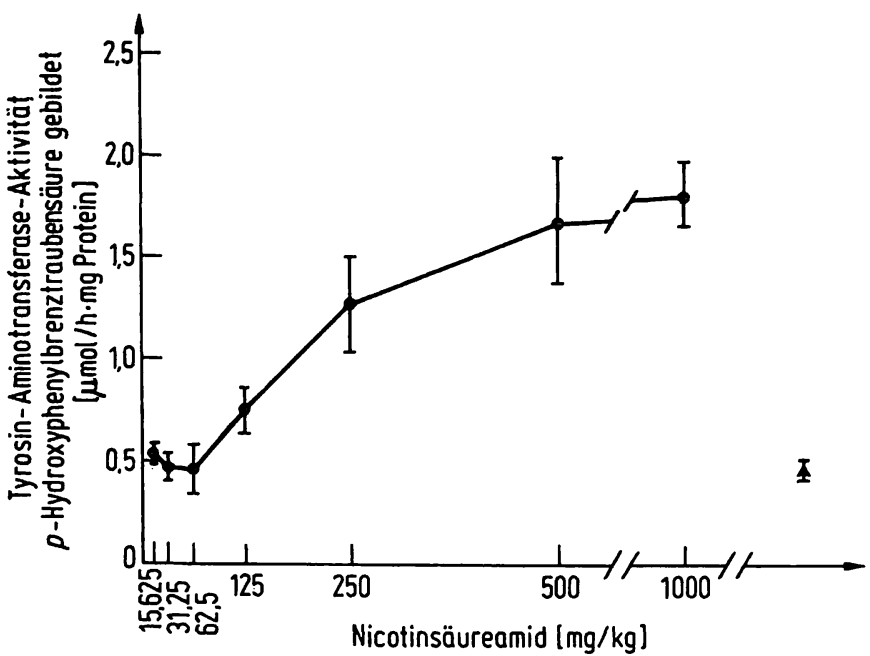

Abb. 6. Einfluß von steigenden Dosen Nicotinsäureamid auf die Aktivität der Tyrosin-Aminotransferase in der Leber normal ernährter, adrenalek tomierter Ratten bei gleichzeitiger Gabe von $2,5 \mathrm{mg} / \mathrm{kg}$ Cortisonacetat. Die Tiere wurden 9 Stunden nach der Injektion getötet.

$\overline{\mathrm{x}} \pm s_{\overline{\mathrm{x}}} ; \mathrm{n}=\mathrm{je} 3-4$ Tiere

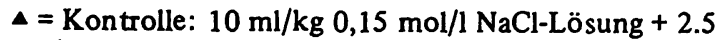
$\mathrm{mg} / \mathrm{kg}$ Cortisonacetat; signifikant unterschiedlich bei $5 \%$ Irrtumswahrscheinlichkeit $\mathrm{ab} \geqq 125 \mathrm{mg} / \mathrm{kg}$ Nicotinsäureamid

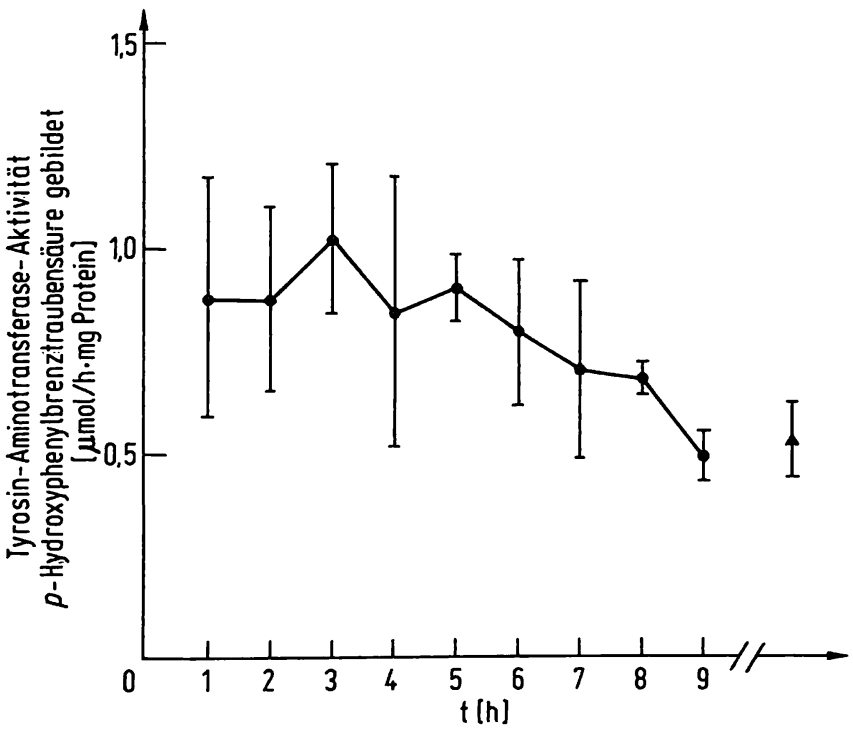

Abb. 7. Induktion der Tyrosin-Aminotransferase durch $500 \mathrm{mg} / \mathrm{kg}$ $L$-Methionin in der Leber normal ernährter, adrenalek tomierter Ratten in Abhängigkeit von der Zeit. $\overline{\mathbf{x}} \pm s_{\overline{\mathbf{x}}} ; \mathrm{n}=$ je 3 Tiere

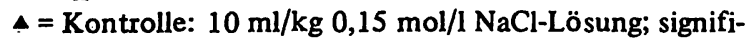
kant unterschiedlich bei $5 \%$ Irrtumswarscheinlichkeit bei $\leqq 6 h, 8 h$

Aminotransferase, wenn auch nur gering, angestiegen ist. Von der 3. Stunde an kommt es dann zu einem steten Abfall der Tyrosin-Aminotransferase-Aktivität.

Nicotinsäureamid +L-Methionin: Wenn steigenden Dosẹn Niçotinsäureamid jeweils $300 \mathrm{mg} / \mathrm{kg} L$-Methionin 


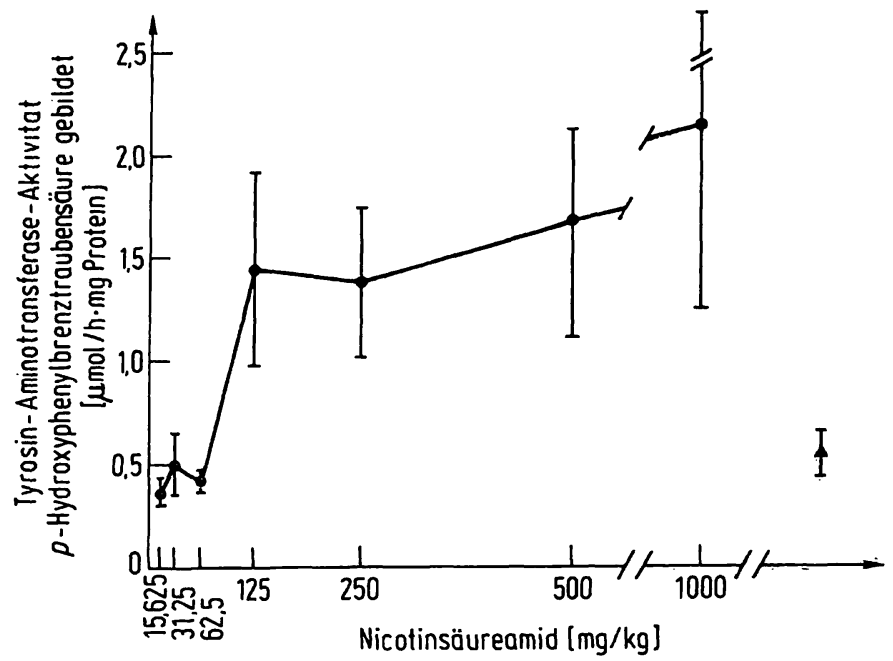

Abb. 8. Einfluß von steigenden Dosen Nicotinsäureamid auf die Aktivität der Tyrosin-Aminotransferase in der Leber normal ernährter, adrenalek tomierter Ratten bei gleichzeitiger Gabe von $300 \mathrm{mg} / \mathrm{kg} L$-Methionin. Die Tiere wurden 9 Stunden nach der Injektion getötet.

$\overline{\mathrm{x}} \pm \mathrm{s}_{\overline{\mathrm{x}}} ; \mathrm{n}=\mathrm{je} 4$ Tiere

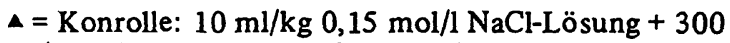
$\mathrm{mg} / \mathrm{kg} L$-Methionin; signifikant unterschiedlich bei $5 \%$ Irrtumswahrscheinlichkeit $a b \geqq 125 \mathrm{mg} / \mathrm{kg}$ Nicotinsäureamid

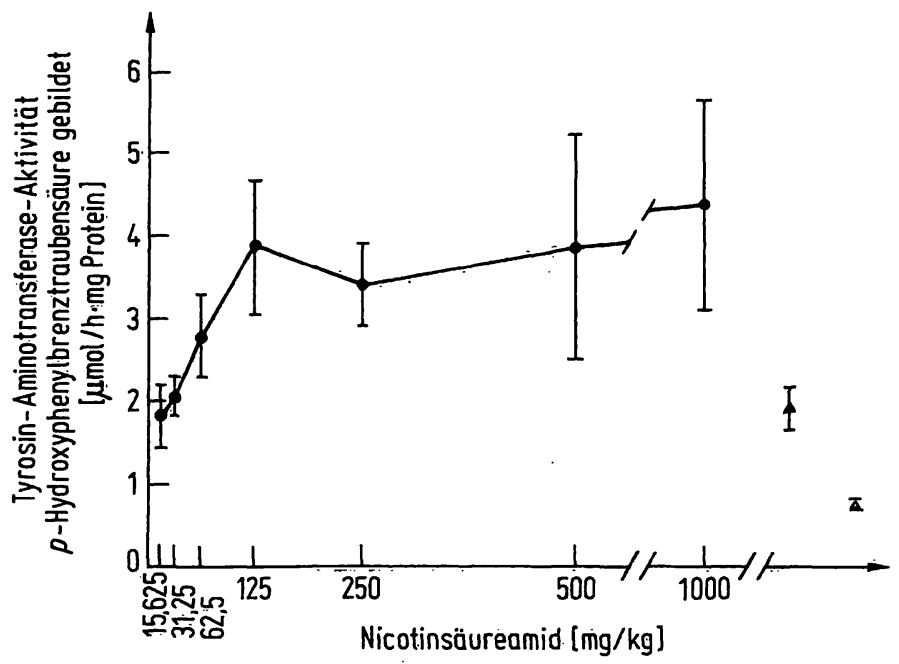

Abb. 10. Einfluß von steigenden Dosen Nicotinsäureamid auf die Aktivität der Tyrosin-Aminotransferase in der Leber normal ernährter Ratten bei gleichzeitiger Gabe von $2,5 \mathrm{mg} / \mathrm{kg}$ Cortisonacetat, $300 \mathrm{mg} / \mathrm{kg} L$-Methionin und $400 \mathrm{mg} / \mathrm{kg} L$-Tyrosin. Die Tiere wurden 9 Stunden nach der Injektion getötet.

$\ddot{\bar{x}} \pm s_{\bar{x}} ; n=j e 6$ Tiere

$\Delta=$ Kontrolle I: $10 \mathrm{ml} / \mathrm{kg} 0,15 \mathrm{~mol} / 1 \mathrm{NaCl}$-Lösung + $300 \mathrm{mg} / \mathrm{kg} L$-Methionin $+2,5 \mathrm{mg} / \mathrm{kg}$ Cortisonacetat + $400 \mathrm{mg} / \mathrm{kg} L$-Tyrosin; signifikant unterschiedlich ab $\geqq 62,5 \mathrm{mg} / \mathrm{kg}$ Nicotinsäureamid

$\Delta=$ Kontrolle II: $10 \mathrm{ml} / \mathrm{kg} 0,15 \mathrm{~mol} / 1 \mathrm{NaCl}$-Lösung; signifikant unterschiedlich bei $5 \%$ Irrtumswahrscheinlichkeit $\mathrm{ab} \geqq 15,625 \mathrm{mg} / \mathrm{kg}$ Nicotinsäureamid

zugesetzt werden, tritt nur eine geringe Zunahme der Tyrosin-Aminotransferase-Aktivität ein (Abb. 8).

Nicotinsäureamid + L-Methionin + Cortisonacetat: Bei dieser Kombination liegt die Tyrosin-AminotransferaseAktivität am höchsten bei einer Dosis von $500 \mathrm{mg} / \mathrm{kg}$ Nicotinsäureamid (Abb. 9).

Nicotinsäureamid + L-Methionin + Cortisonacetat + I I Tyrosin-Aminotransferase-Aktivität die von allen Versuchen höchsten Werte (Abb. 10). Dieses zeigt sich schon bei geringen Dosen Nicotinsäureamid.

\section{Diskussion}

Die Induktion der Tyrosin-Aminotransferase durch Nicotinsäureamid wird erheblich gesteigert, wenn das Nicotinsäureamid in einer Kombination mit $L$-Methionin, Cortisonacetat und $L$-Tyrosin verabreicht wird. Nicotinsäureamid ohne Zusätze führt zu einer wesentlich geringeren Steigerung; dieses ist bei adrenalektomierten Tieren noch stärker ausgeprägt als bei intakten. Bei den Enzymen Transketolase und Transaldolase hat man ähnliche Ergebnisse gefunden (9): Daraus läßt sich ableiten, daß das Cortison für die Induktion eine Rolle spielt. 
Die Ursache für die durch Nicotinsäureamid gesteigerte Enzym-Induktion könnte darin begründet liegen, daß das applizierte Nicotinsäureamid eine Zunahme des Nicotinsäureamidpools bewirkt $(13,14)$ und als Substrat u. a. die Bildung der Polyadenosindiphosphat-ribose begünstigt, deren Beteiligung bei Regulations- bzw. Differenzierungsprozessen diskutiert wird.

\section{Literatur}

1. Kröger, H., Löwel, M. \& Kessel, H. (1968), Hoppe-Seyler's Z. Phy siol. Chem. 349, 1221-1224.

2. Rosen, F. \& Milholland, R. J. (1963), J. Biol. Chem. 238 , 3730-3735.

3. Blake, R. L., Blake, S. L., Loh, H. H. \& Kun, E. (1967), Mol. Pharmakol. 3, 412-422.

4. Blake, R. L. (1970), Can. J. Biochem. 48, 1043-1049.

5. Blake, R. L. \& Kun, E. (1971), Methods Enzymology 18, 113-123.

6. Yamaguchi, K., Sakakibara, S., Kogy, K. \& Neda, I. (1971), Biochim. Biophys. Acta 237, 502-512.

7. Pallini, V., Vasconetto, C. \& Ricci, C. (1965), Giorn. Biochim. 14, 743-748.

8. Labbe, R. F., Nutter, J., Carger, M. L. \& Nielsen, L. C. (1970), Biochem. Med. 3, 465-474.

9. Lorenzoni, I., Calabria, G. A. \& DeFlora, A. (1966), Boll. Soc. Ital. Biol. 42, 899-902.

10. Kröger, H. \& Greuer, B. (1965), Biochem. Z. 341, 190-198.

11. Gornall, A. G., Bardawill, C. J. \& David, M. M. (1949), J. Biol. Chem. 177, 751-764.

12. Kröger, H. \& Greuer, B. (1966), Nature 210, 200-201.

13. Feigelson, P., Williams jr, J. N. \& Elvehjem, C. A. (1951), Proc. Soc. Exp. Biol. Med. 78, 34-36.

14. Kröger, I., Werchau, H. \& Kröger, H. (1966), Z. Krebsforsch. $68,156-163$.
Prof. Dr. Dr. H. Kröger Robert Koch-Institut Nordufer 20 1000 Berlin 65 
.

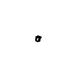

